\title{
Note sulla distribuzione in Italia di Anoxia (Mesanoxia) matutinalis matutinalis Laporte de Castelnau, 1832 con nuovi dati corologici per alcune regioni (Coleoptera, Melolonthidae)
}

Riassunto - Viene fornita una panoramica generale della distribuzione in Italia di Anoxia (Mesanoxia) matutinalis matutinalis Laporte de Castelnau, 1832, aggiungendo Lombardia e Umbria alle regioni in cui questa specie è riportata come presente in Italia. Vengono inoltre rese note le prime segnalazioni per il Lazio settentrionale.

Parole chiave: Anoxia matutinalis matutinalis, Melolonthidae, distribuzione, Italia.

\begin{abstract}
Notes on the distribution in Italy of Anoxia (Mesanoxia) matutinalis matutinalis Laporte de Castelnau, 1832 with new chorological data for some regions (Coleoptera, Melolonthidae).

A generale overview of the Italian distribution of Anoxia (Mesanoxia) matutinalis matutinalis Laporte de Castelnau, 1832 is given, including new records for Lombardy and Umbria. Moreover, new records for northern Latium are reported.
\end{abstract}

Key words: Anoxia matutinalis matutinalis, Melolonthidae, distribution, Italy.

\section{Introduzione}

Anoxia (Mesanoxia) matutinalis Laporte de Castelnau, 1832 è una delle sei specie della fauna italiana appartenenti al genere Anoxia. Ben riconoscibile per una vistosa pubescenza elitrale riunita a formare fasce longitudinali intere e ordinate (Ballerio et al., 2010), A. matutinalis è una specie politipica a corotipo S-europeo, che comprende sei sottospecie, molte delle quali presenti esclusivamente in territori ristretti: A. m. suturalis Reitter 1890, conosciuta esclusivamente per la Grecia (Bezdek, 2006), A. m. moltonii Sabatinelli, 1976, endemica dell'Isola di Vulcano nelle Eolie (Sabatinelli, 1976; Ballerio et al., 2010), A. m. corsicana Sabatinelli, 
1976, endemica della Corsica e della Sardegna settentrionale (cfr. Sabatinelli, 1976; Pesarini, 2004; Ballerio et al., 2010), A. m. sardoa Motschulsky, 1860, endemica della Sardegna centro-meridionale (cfr. Pesarini, 2004; Ballerio et al., 2010; Guerlach, 2012), A. m. thumseri Guerlach, 2011, recentemente scoperta nella provincia di Oristano in Sardegna (Guerlach, 2011, 2012) e A. m. matutinalis Laporte de Castelnau, 1832, che risulta quella a più ampia distribuzione, dall'Italia (Sicilia inclusa) alle coste adriatiche della penisola balcanica e a Malta (cfr. Sabatinelli, 1976; Sabatinelli \& Schembri, 1990; Bezdek, 2006; Ballerio et al., 2010; Pivotti et al., 2011). La sottospecie nominale, legata perlopiù ad ambienti costieri e terreni sabbiosi, in Italia è presente in buona parte del territorio, essendo segnalata per Liguria, Toscana, Abruzzo, Molise, Campania, Puglia, Basilicata, Calabria e Sicilia (Ballerio et al., 2010), Emilia-Romagna (Zangheri, 1969; Cerretti et al., 2003; Ente per i Parchi e la Biodiversità Emilia Orientale, 2012), Friuli-Venezia Giulia (Sabatinelli, 1976; Pesarini, 2004; Ballerio et al., 2011), Veneto (Pesarini, 2004; Ballerio et al., 2011), Trentino (Halbherr, 1892, 1908) e Alto Adige (Peez \& Kahler, 1977; Hellrigl, 1996). Per il Lazio esiste una segnalazione di due esemplari raccolti nell'isola di Palmarola, nell'arcipelago delle Isole Ponziane (Carpaneto, 1979); per il resto della regione, in cui A. m. matutinalis è indicata genericamente come presente da Luigioni (1929), Porta (1934) e Ballerio et al. (2011), sono riportate indicazioni di località soltanto per i dintorni di Roma (Luigioni, 1905; Carpaneto \& Piattella, 1997; Carpaneto et al., 1997).

Con il presente contributo vengono forniti dati corologici inediti per Anoxia matutinalis matutinalis, aggiungendo alcune nuove regioni al suo areale di diffusione in Italia e specificandone meglio la distribuzione per un'altra.

\section{Materiali e metodi}

Il materiale utilizzato per il presente contributo proviene dalle collezioni degli autori e del Museo di Storia Naturale dell'Università di Firenze, sezione di Zoologia "La Specola". Tutti gli esemplari sono stati determinati o rivisti dagli autori. Per ciascun lotto sono riportati i dati di cattura disponibili, il numero degli esemplari e la collezione di appartenenza.

Le abbreviazioni utilizzate hanno il seguente significato:

$\mathrm{CFB}=$ Collezione Fausto Barbagli, Arezzo;

$\mathrm{CFC}=$ Collezione Filippo Ceccolini, Rassina (Arezzo);

MSNF $=$ Museo di Storia Naturale di Firenze, sez. di Zoologia "La Specola".

\section{Risultati}

Le regioni in cui la presenza di A. matutinalis matutinalis non era precedentemente nota e in cui essa è segnalata per la prima volta sono le seguenti:

Lombardia: Bergamo, dall'Ispettore Forestale, 1 es., MSNF ( $\mathrm{n}^{\circ}$ coll. 16610); dintorni di Milano, [ante 1936], ex coll. E. Turati, 2 es., MSNF ( ${ }^{\circ}$ coll. 16611); Pavia (Palazzo Botta), VII.2003, legit F. Barbagli, 1 es., CFB.

Umbria: Lippiano, Monte Santa Maria Tiberina, Perugia, VIII.1921, ex coll. A. Andreini, 1 es., MSNF ( ${ }^{\circ}$ coll. 16612); idem, VII.1923, 1 es., MSNF ( ${ }^{\circ}$ coll. 16613); idem, 3.VII.1924, 5 es., MSNF ( $\mathrm{n}^{\circ}$ coll. 16614); idem, VII.1924, 7 es., MSNF ( $\mathrm{n}^{\circ}$ coll. 16615); idem, VII.1925, 1 es., MSNF ( $\mathrm{n}^{\circ}$ coll. 16616); idem, VII.1926, 3 es., MSNF ( $\mathrm{n}^{\circ}$ coll. 16617); idem, VI.1927, 4 es., MSNF ( ${ }^{\circ}$ coll. 
16618); idem, VII.1927, 4 es., MSNF ( ${ }^{\circ}$ coll. 16619); idem, VII.1928, 1 es., MSNF $\left(\mathrm{n}^{\circ}\right.$ coll. 16620); idem, VII.1929, 2 es., MSNF ( ${ }^{\circ}$ coll. 16621); idem, VIII.1929, 1 es., MSNF ( $\mathrm{n}^{\circ}$ coll. 16622); idem, VII.1931, 3 es., MSNF ( ${ }^{\circ}$ coll. 16623); idem, VII.1933, 1 es., MSNF ( $\mathrm{n}^{\circ}$ coll. 16624); idem, 5.VII.1934, 1 es., MSNF ( ${ }^{\circ}$ coll. 16625); idem, VII.1934, 1 es., MSNF ( ${ }^{\circ}$ coll. 16626); idem, VII.1936, 3 es., MSNF ( ${ }^{\circ}$ coll. 16627); idem, VII.1939, 1 es., MSNF ( ${ }^{\circ}$ coll. 16628); Castiglion del Lago, Perugia, 9.VII.1994, leg. F. Barbagli e S. Goti, 2 es., 1 CFB, 1 CFC.

Sono infine riportati i primi dati per il Lazio settentrionale:

Lazio: Montalto Marina, Montalto di Castro, Viterbo, VII.1992, legit Fb [= Fabio] Barbagli, 1 es., CFB; idem, 18.VII.1995, legit G. Forzoni, 1 es. (Fig. 1a), CFB; idem, 4.VII.1998, leg. Fb e Fl [= Filippo] Barbagli, 1 es. (Fig. 1b), CFC; idem, 18.VII.1998, 1 es., leg. Fb e Fl Barbagli, CFB.

\section{Discussione}

Gli esemplari menzionati in questo contributo costituiscono le prime segnalazioni di Anoxia matutinalis matutinalis per la Lombardia e l'Umbria, portando così a 16 il numero delle regioni in cui tale sottospecie è segnalata in Italia. Inoltre vengono forniti i primi dati per il Lazio settentrionale. Con le segnalazioni per Umbria e Lazio viene documentata meglio la continuità dell'areale del taxon nell'Italia centrale, mentre quelle lombarde permettono di ampliare a nord-ovest tale areale. Queste segnalazioni dimostrano come le informazioni relative alla corologia di
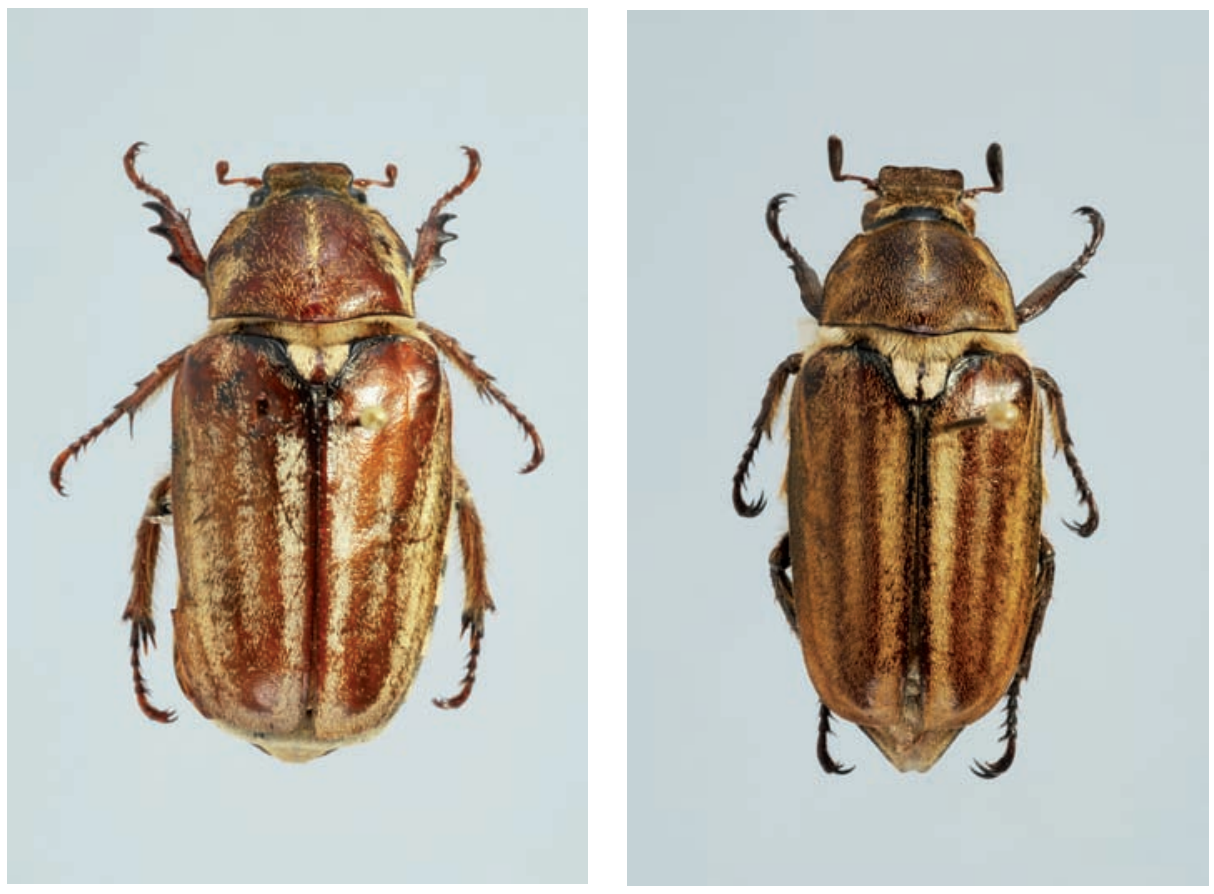

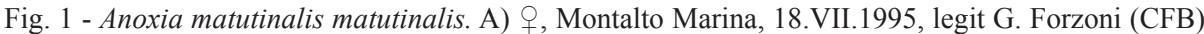
B) $\curvearrowright$, 4.VII.1998, leg. Fb e Fl Barbagli (CFC). (Foto/Photo by: S. Bambi). 
A. matutinalis matutinalis fossero ancora piuttosto incomplete, nonostante l'aspetto vistoso e la relativa facilità di reperimento di questo coleottero. È peraltro possibile che l'areale possa essere ulteriormente esteso grazie ad una verifica dettagliata del materiale conservato nelle collezioni dei vari Musei. Alla luce dei dati riportati è in ogni caso ipotizzabile una diffusione ancora più estesa di questa sottospecie in Italia, in quanto appare plausibile la sua presenza anche in Piemonte e nelle Marche, che presentano, oltre che una collocazione limitrofa all'areale conosciuto, anche ambienti favorevoli per la biologia di A. matutinalis. Per quanto riguarda le Marche, va però sottolineato come in uno studio frutto di un'attività di ricerca effettuata sulla coleotterofauna della regione tra il 1987 e il 2012, A. matutinalis matutinalis non sia mai stata reperita (Giovagnoli et al., 2012). In generale comunque, ulteriori ricerche sarebbero auspicabili per incrementare i dati corologici relativi a questo melolontide, non solo per ottenere informazioni dall'intrinseco valore faunistico, ma anche per monitorare possibili effetti sulla vegetazione, come è stato fatto attraverso studi condotti in Salento che dimostrano un forte impatto sulle pinete di Pinus halepensis Miller e P. pinea Linnaeus, vittime di grandi defogliazioni da parte degli adulti di A. matutinalis matutinalis (Triggiani \& Tarasco, 2002; Tarasco \& Triggiani, 2011).

\section{Ringraziamenti}

Desideriamo ringraziare vivamente Luca Bartolozzi del Museo di Storia Naturale dell'Università di Firenze, sezione di Zoologia "La Specola" per averci consentito di esaminare il materiale museale sotto la sua cura e per avere cortesemente riletto il manoscritto; Carlo Violani per gli utili suggerimenti; Antonio Rey per le preziose informazioni che ha gentilmente fornito; Fernando Pederzani per la cortesia mostrata nel fornirci celermente materiale bibliografico richiesto; Saulo Bambi per le fotografie riportate nel testo; i referee anonimi per i costruttivi commenti.

\section{Bibliografia}

Ballerio A., Rey A., Uliana M., Rastelli M., Rastelli S., Romano M. \& Colacurcio L., 2010 - Coleotteri Scarabeoidei d'Italia. Piccole Faune. DVD.

Ballerio A., Rey A., Uliana M., Rastelli M., Rastelli S., Romano M. \& Colacurcio L., 2011 - Coleotteri Scarabaeoidei d'Italia. Aggiunte e Correzioni. - [electronic publication].

Bezdek A., 2006 - Scarabaeidae, Melolonthinae, Melolonthini. In: Catalogue of Palaearctic Coleoptera. Vol. 3. Löbl I. \& Smetana A. (eds). Apollo Books, Stenstrup: 191-198.

Carpaneto G.M., 1979 - Osservazioni sui Coleotteri Scarabeoidea delle isole Ponziane. Fragmenta Entomologica, Roma, 15 (1): 111-126.

Carpaneto G.M. \& Piattella E., 1997 - Coleoptera Lucanoidea e Scarabaeoidea, pp. 154-165. In: Gli Insetti di Roma. Zapparoli, M. (ed.). Fratelli Palombi Editori, Roma.

Carpaneto G.M., Maltzeff P., Piattella E. \& Pontuale G., 1997 - I Coleotteri lamellicorni della tenuta presidenziale di Castelporziano e delle aree limitrofe (Coleoptera, Lamellicornia). Bollettino dell'Associazione Romana di Entomologia, Roma, 52 (1-4): 9-54. 
Cerretti P., Tagliapietra A., Tisato M., Vanin S., Mason F. \& Zapparoli M. (a cura di), 2003 - Artropodi dell'orizzonte del faggio nell'Appennino settentrionale. Gianluigi Arcari Editore, Mantova.

Ente per i Parchi e la Biodiversità Emilia Orientale, 2012 - Misure Specifiche di Conservazione - Piani di Gestione dei Siti Natura 2000. Quadro conoscitivo parte seconda.

http:/www.enteparchi.bo.it/pdf/sic-corno-alle-scale/quadroconoscitivo_2.pdf

Giovagnoli G., Strocchi A. \& Paglialunga M., 2012 - Coleotteri della Regione Marche. Primo contributo alla conoscenza della coleotterofauna della Regione Marche (Insecta Coleoptera Carabidae, Buprestidae, Meloidae, Tenebrionidae, Lucanidae, Bolboceratidae, Melolonthidae, Cetoniidae, Cerambycidae). Quaderno di Studi e Notizie di Storia Naturale della Romagna, Bagnocavallo, 36 : 159-184.

Guerlach G., 2011 - Description d'une nouvelle sous-espèce d'Anoxia matutinalis Castelnau, 1832: Anoxia matutinalis thumseri n. ssp. Lambillionea, Chênée, 111 (3, Tome 1): 249-252.

Guerlach G., 2012 - Description de la femelle d'Anoxia matutinalis thumseri Guerlach, 2011 (Coleoptera, Scarabaeoidea, Melolonthidae, Melolonthinae, Melolonthini). Lambillionea, Chênée, 112 (2): 128-130.

Halbherr B., 1892 - Elenco sistematico dei Coleotteri finora raccolti nella Valle Lagarina. Fascicolo V. Platyceridae-Scarabaeidae. Pubblicazioni del Museo Civico di Rovereto, Rovereto, 21: 1-35.

Halbherr B., 1908 - Aggiunte all'elenco sistematico dei coleotteri fin ora raccolti nella Valle Lagarina. Pubblicazioni del Museo Civico di Rovereto, Rovereto, 45: $1-41$.

Hellrigl K., 1996 - Die Tierwelt Südtirols. Kommentiertes systematisch-faunistisches verzeichnis der auf dem gebiet der provinz Bozen- Südtirol (Italien) bekannten Tierarten. Naturmuseum Südtirol, Bozen.

Luigioni P., 1905 - Coleotteri del Lazio, notati od omessi nel catalogo dei Coleotteri del Dott. Stefano Bertolini. Rivista Coleotterologica Italiana, Camerino, 3: 253-278.

Luigioni P., 1929 - I Coleotteri d'Italia. Catalogo sinonimico-topograficobibliografico. Memorie della Pontificia Accademia delle Scienze - Inuovi Lincei (Serie II), 13.

Peez A. \& Kahlen M., 1977 - Die Käfer von Sud Tirol. Faunistisches Verzeichnis der aus de Provinz Bozen bisher bekannt gewordenen Koleopteren. Im Selbstverlag de Tiroler Landesmuseum Ferdinandeum, Innsbruck, 2: $1-525$.

Pesarini C., 2004 - Insetti della Fauna Italiana. Coleotteri Lamellicorni. Natura. Rivista di Scienze Naturali, Milano, 93 (2): 1-130.

Pivotti I., Agoglitta R., Zunino M., Piattella E., Dellacasa M., Corallini C. \& Mifsud D., 2011 - The Scarabaeoidea of the Maltese Islands (Central Mediterranean) (Coleoptera). Bulletin of the Entomological Society of Malta, Valletta, 4: $85-124$.

Porta A., 1934 - Fauna Coleopterorum Italica. Rhynchophora - Lamellicornia Vol. V. Stabilimento Tipografico Piacentino, Piacenza.

Sabatinelli G., 1976 - Revisione delle specie italiane del sottogenere Mesanoxia Med. (Coleoptera Scarabaeidae, Melolonthidae). Fragmenta Entomologica, Roma, 12: 143-157. 
Sabatinelli G. \& Schembri S., 1990 - Gli scarabeidi floricoli delle isole maltesi. Bollettino della Società entomologica Italiana, Genova, 122 (1): 31-36.

Tarasco E. \& Triggiani O., 2011 - Biological and Morphological Aspects of Anoxia (Mesanoxia) matutinalis ssp. matutinalis Castelnau, 1832 (Coleoptera, Melolonthidae), a Pest of Apulian Pinewoods in Southern Italy. Silva Lusitana, Oeiras, 19 ( $\mathrm{n}^{\mathrm{o}}$ especial): 75-78.

Triggiani O. \& Tarasco E., 2002 - Ingenti attacchi del coleottero scarabeide Anoxia (Mesanoxia) matutinalis ssp. matutinalis Castelnau, 1832 nelle pinete del Salento. Atti del XIX Congresso Nazionale Italiano di Entomologia, Catania: 339-343.

Zangheri P., 1969 - Repertorio sistematico e topografico della flora e fauna vivente e fossile della Romagna. Tomo III. Museo Civico di Storia Naturale di Verona. Memorie fuori serie N. 1, Verona.

Ricevuto: 19 marzo 2013

Approvato: 18 aprile 2013 